\title{
On the boolean minimal realization problem in the max-plus algebra
}

\author{
Bart De Schutter $^{\mathrm{a}, *}$, Vincent Blondel ${ }^{\mathrm{b}}$, Remco de Vries ${ }^{\mathrm{a}}$, Bart De Moor ${ }^{\mathrm{a}}$ \\ a ESAT-SISTA, K.U. Leuven, Kardinaal Mercierlaan 94, B-3001 Heverlee (Leuven), Belgium \\ b Institute of Mathematics, University of Liège, Sart Tilman B37, B-4000 Liège, Belgium \\ Received 18 December 1997; received in revised form 5 March 1998; accepted 25 March 1998
}

\begin{abstract}
One of the open problems in the max-plus-algebraic system theory for discrete event systems is the minimal realization problem. In this paper we present some results in connection with the minimal realization problem in the max-plus algebra. First we characterize the minimal system order of a max-linear discrete event system. We also introduce a canonical representation of the impulse response of a max-linear discrete event system. Next we consider a simplified version of the general minimal realization problem: the boolean minimal realization problem, i.e., we consider models in which the entries of the system matrices are either equal to the max-plus-algebraic zero element or to the max-plus-algebraic identity element. We give a lower bound for the minimal system order of a max-plus-algebraic boolean discrete event system. We show that the decision problem that corresponds to the boolean realization problem (i.e., deciding whether or not a boolean realization of a given order exists) is decidable, and that the boolean minimal realization problem can be solved in a number of elementary operations that is bounded from above by an exponential of the square of (any upper bound of) the minimal system order. We also point out some open problems, the most important of which is whether or not the boolean minimal realization problem can be solved in polynomial time. (c) 1998 Elsevier Science B.V. All rights reserved.
\end{abstract}

Keywords: Discrete event systems; Max-plus algebra; Minimal state space realization; Boolean max-plus algebra; Complexity

\section{Introduction}

The max-plus-algebra $[1,4]$, which has maximization and addition as its basic operations, is one of the frameworks that can be used to model a class of discrete event systems (DESs). Typical examples of DESs are flexible manufacturing systems, telecommunication networks, parallel processing systems and logistic systems. One of the characteristic features of DESs, as opposed to continuous variable systems (i.e., systems the behavior of which can be described by difference or differential equations), is that their dynamics are event-driven as opposed to time-driven.

* Corresponding author. Tel.: +32-16-32.17.09; fax: +32-1632.19.70; e-mail: bart.deschutter@esat.kuleuven.ac.be.
An event corresponds to the start or the end of an activity. For a manufacturing system possible events are: the completion of a part on a machine, a machine breakdown, or a buffer becoming empty.

In general, models that describe the behavior of a DES are nonlinear, but there exists a class of DESs - the max-linear DESs - for which the model becomes "linear" when formulated in the max-plus algebra $[1,3,4]$. One of the open problems in the max-plus-algebraic system theory for DESs is the minimal realization problem, which can be stated as follows: given the impulse response of a max-linear DES, determine a model of smallest possible size the impulse response of which coincides with the given impulse response. The minimal realization problem in the max-plus algebra is the central topic of this paper. 


\section{Preliminaries}

\subsection{Notation}

Let $A$ be an $m \times n$ matrix. Then $A_{i, \text {, is }}$ ith row of $A$ and $A_{., j}$ is the $j$ th column of $A$. Let $\alpha \subseteq\{1,2, \ldots, m\}$ and $\beta \subseteq\{1,2, \ldots, n\}$. The submatrix of $A$ obtained by removing all rows of $A$ that are not indexed by $\alpha$ and all columns that are not indexed by $\beta$ is denoted by $A_{\alpha \beta}$. The submatrix of $A$ obtained by removing all rows (columns) of $A$ except for those indexed by $\alpha(\beta)$ is denoted by $A_{\alpha, .}\left(A_{., \beta}\right)$.

If $x \in \mathbb{R}$ then $\lceil x\rceil$ is the smallest integer that is larger than or equal to $x$. Two real functions $f$ and $g$ are asymptotically equivalent in the neighborhood of $\infty$, denoted by $f(x) \sim g(x), x \rightarrow \infty$, if $\lim _{x \rightarrow \infty} f(x) / g(x)=1$. The set of the nonnegative (positive) integers is denoted by $\mathbb{N}\left(\mathbb{N}_{0}\right)$.

\subsection{Max-plus algebra}

Define $\varepsilon=-\infty$ and $\mathbb{R}_{\varepsilon}=\mathbb{R} \cup\{\varepsilon\}$. The basic operations of the max-plus algebra $[1,4]$ are the maximum (represented by $\oplus$ ) and the addition (represented by $\otimes)$ :

$x \oplus y=\max (x, y), \quad x \otimes y=x+y$

with $x, y \in \mathbb{R}_{\varepsilon}$. We call $\oplus$ the max-plus-algebraic sum and $\otimes$ the max-plus-algebraic product. Note that $\varepsilon$ and 0 are the identity elements for respectively $\oplus$ and $\otimes$.

Remark 2.1. The analogy between $\oplus$ and + , and between $\otimes$ and $\times$ is evidenced by the following equivalences:

$x \oplus y=z \Leftrightarrow \mathrm{e}^{x s}+\mathrm{e}^{y s} \sim c \mathrm{e}^{z s}, \quad s \rightarrow \infty$,

$x \otimes y=z \Leftrightarrow \mathrm{e}^{x s} \cdot \mathrm{e}^{y s}=\mathrm{e}^{z s}$ for all $s>0$

with $x, y, z \in \mathbb{R}_{\varepsilon}$, and $c=2$ if $x=y$ and $c=1$ otherwise. ${ }^{1}$ Using this transformation the structure $\left(\mathbb{R}_{\varepsilon}, \oplus, \otimes\right)$ can be mapped to a structure consisting of exponentials with conventional addition and multiplication as basic operations (see $[16,17]$ ). Note that the exponential transformation maps $\varepsilon$ to 0 , and 0 to 1 . This mapping allows us to transform some results from conventional algebra to the max-plus algebra. However, since there does not exist an equivalent of the minus operator in the max-plus algebra,

\footnotetext{
${ }^{1} \mathrm{We}$ assume that $\mathrm{e}^{\varepsilon s}=0$ for all $s>0$ by definition.
}

many results and techniques of conventional algebra and linear system theory cannot be translated in a straightforward way to the max-plus algebra and max-plus-algebraic system theory.

However, if we restrict ourselves to results in "nonnegative" linear algebra (i.e., results in which the minus and the subtraction operators do not appear), then we can transform these results to the max-plus algebra. This makes that many results of, e.g., linear system theory for nonnegative systems can be transformed to max-plus-algebraic system theory and vice versa (see also Section 3.2 and the paragraph before Proposition 3.4). As a consequence, the problems discussed in this paper are not only relevant to the discrete event systems domain but to other domains - such as linear system theory for nonnegative systems - as well.

The operations $\oplus$ and $\otimes$ are extended to matrices as follows. If $A, B \in \mathbb{R}_{\varepsilon}^{m \times n}$ then

$$
(A \oplus B)_{i j}=a_{i j} \oplus b_{i j}
$$

for all $i, j$. If $A \in \mathbb{R}_{\varepsilon}^{m \times p}$ and $B \in \mathbb{R}_{\varepsilon}^{p \times n}$ then

$$
(A \otimes B)_{i j}=\bigoplus_{k=1}^{p} a_{i k} \otimes b_{k j}
$$

for all $i, j$. The matrix $\varepsilon_{m \times n}$ is the max-plus-algebraic zero matrix: $\left(\varepsilon_{m \times n}\right)_{i j}=\varepsilon$ for all $i, j$. If the dimensions of the max-plus-algebraic zero matrix are not indicated, they should be clear from the context. The matrix $E_{n}$ is the max-plus-algebraic identity matrix: we have $\left(E_{n}\right)_{i i}=0$ for all $i$ and $\left(E_{n}\right)_{i j}=\varepsilon$ for all $i, j$ with $i \neq j$. The $k$ th max-plus-algebraic matrix power of a matrix $A \in \mathbb{R}_{\varepsilon}^{n \times n}$ with $k \in \mathbb{N}$ is defined as follows:

$$
A^{\otimes^{0}}=E_{n} \quad \text { and } \quad A^{\otimes^{k}}=\underbrace{A \otimes A \otimes \cdots \otimes A}_{k \text { times }} \text { if } k>0 .
$$

Define $\mathbb{B}=\{0, \varepsilon\}$. A matrix with entries in $\mathbb{B}$ is called a max-plus-algebraic boolean matrix.

\subsection{Graph theory}

In order to define some additional max-plusalgebraic concepts and to prove some propositions in the next sections, we also need some results from graph theory, which will be presented in this section.

A graph $\mathscr{G}$ is defined as an ordered pair $(V, E)$, where $V$ is a set of elements called vertices and $E$ is a set of (unordered) pairs of vertices. The elements of $E$ are called edges. 
If the vertices of a graph can be partitioned into two disjunct sets $X$ and $Y$ such that all edges go from vertices in $X$ to vertices in $Y$, then the graph is called bipartite. Consider a bipartite graph $\mathscr{G}=(X \cup Y, E)$ with $X=\left\{x_{1}, x_{2}, \ldots, x_{m}\right\}, Y=\left\{y_{1}, y_{2}, \ldots, y_{n}\right\}, X \cap Y=\emptyset$ and such that every element of $E$ can be written as $\{x, y\}$ with $x \in X$ and $y \in Y$. With $\mathscr{G}$ we associate a matrix $A \in \mathbb{B}^{n \times m}$ by setting $a_{j i}=0$ if there is an edge between $x_{i}$ and $y_{j}$ and $a_{j i}=\varepsilon$ otherwise. We call $A$ the incidence matrix of $\mathscr{G}$, and $\mathscr{G}$ the transition graph of $A$. If for each $x_{i} \in X$ and each $y_{j} \in Y$, there is an edge between $x_{i}$ and $y_{j}$ then we say that the bipartite graph is complete.

A directed graph $\mathscr{G}$ is defined as an ordered pair $(V, \mathscr{A})$, where $V$ is a set of vertices and $\mathscr{A}$ is a set of ordered pairs of vertices. The elements of $\mathscr{A}$ are called arcs. Let $\mathscr{G}=(V, \mathscr{A})$ be a directed graph with $V=\left\{v_{1}, v_{2}, \ldots, v_{n}\right\}$. A path of length $l\left(l \in \mathbb{N}_{0}\right)$ is a sequence of vertices $v_{i_{0}}, v_{i_{1}}, \ldots, v_{i_{l}}$ such that $\left(v_{i_{k}}, v_{i_{k+1}}\right) \in \mathscr{A}$ for $k=0,1, \ldots, l-1$. We represent this path by $v_{i_{0}} \rightarrow v_{i_{1}} \rightarrow \cdots \rightarrow v_{i_{l}}$. Vertex $v_{i_{0}}$ is the initial vertex of the path and $v_{i_{l}}$ is the final vertex of the path. When the initial and the final vertex of a path coincide, we have a circuit. An elementary circuit is a circuit in which no vertex appears more than once, except for the initial vertex, which appears exactly twice.

A directed graph $\mathscr{G}=(V, \mathscr{A})$ is called strongly connected if for any two different vertices $v_{i}, v_{j} \in V$ there exists a path from $v_{i}$ to $v_{j}$. A maximal strongly connected subgraph (m.s.c.s.) $\mathscr{G}_{\text {sub }}$ of a directed graph $\mathscr{G}$ is a strongly connected subgraph that is maximal, i.e., if we add any extra vertex (and the corresponding arcs) of $\mathscr{G}$ to $\mathscr{G}_{\text {sub }}$ then $\mathscr{G}_{\text {sub }}$ is no longer strongly connected.

The cyclicity $^{2}$ of an m.s.c.s. is the greatest common divisor of the lengths of all the circuits of the given m.s.c.s. If an m.s.c.s. or a graph contains no circuits then its cyclicity is equal to 0 by definition. The cyclicity $c(\mathscr{G})$ of a graph $\mathscr{G}$ is the least common multiple of the nonzero cyclicities of its m.s.c.s.'s.

If we have a directed graph $\mathscr{G}=(V, \mathscr{A})$ with $V=\{1,2, \ldots, n\}$ and if we associate a real number $a_{i j}$ with each arc $(j, i) \in \mathscr{A}$, then we say that $\mathscr{G}$ is a weighted directed graph. We call $a_{i j}$ the weight of the $\operatorname{arc}(j, i)$. Note that the first subscript of $a_{i j}$ corresponds to the final (and not the initial) vertex of the $\operatorname{arc}(j, i)$.

${ }^{2}$ This definition of cyclicity has been introduced in [2] (see also [1]).
Consider $A \in \mathbb{R}_{\varepsilon}^{n \times n}$. The precedence graph of $A$, denoted by $\mathscr{G}(A)$, is a weighted directed graph with set of vertices $\{1,2, \ldots, n\}$ and an $\operatorname{arc}(j, i)$ with weight $a_{i j}$ for each $a_{i j} \neq \varepsilon$. The weight of a path $i_{1} \rightarrow i_{2} \rightarrow \cdots \rightarrow i_{l}$ in $\mathscr{G}(A)$ is defined as the sum of the weights of the arcs that compose the path: $a_{i_{2} i_{1}}+a_{i_{3} i_{2}}+\cdots+a_{i_{l} i_{l-1}}$. The average weight of a circuit is defined as the weight of the circuit divided by the length of the circuit. An elementary circuit of $\mathscr{G}(A)$ is called critical if it has maximum average weight among all circuits. The critical graph $\mathscr{G}^{\mathrm{c}}(A)$ consists of those vertices and $\operatorname{arcs}$ of $\mathscr{G}(A)$ that belong to a critical circuit of $\mathscr{G}(A)$.

The cyclicity of a matrix $A \in \mathbb{R}_{\varepsilon}^{n \times n}$ is denoted by $c(A)$ and is equal to the cyclicity of the critical graph of the precedence graph of $A$. So $c(A)=c\left(\mathscr{G}^{\mathrm{c}}(A)\right)$. Note that if $A \in \mathbb{B}^{n \times n}$ then every circuit in $\mathscr{G}(A)$ is critical, which implies that $c(A)=c\left(\mathscr{G}^{\mathrm{c}}(A)\right)=c(\mathscr{G}(A))$.

\subsection{Some extra definitions and propositions}

Definition 2.2 (Irreducibility). The matrix $A \in \mathbb{R}_{\varepsilon}^{n \times n}$ with $n \geqslant 2$ is called irreducible if $\mathscr{G}(A)$ is strongly connected, i.e., if $\left(A \oplus A^{\otimes^{2}} \oplus \cdots \oplus A^{\otimes^{n-1}}\right)_{i j} \neq \varepsilon$ for all $i, j$ with $i \neq j$. By definition a $1 \times 1$ matrix is always irreducible.

Definition 2.3 (Max-plus-algebraic eigenvalue and eigenvector). Let $A \in \mathbb{R}_{\varepsilon}^{n \times n}$. If there exist $\lambda \in \mathbb{R}_{\varepsilon}$ and $v \in \mathbb{R}_{\varepsilon}^{n}$ with $v \neq \varepsilon_{n \times 1}$ such that $A \otimes v=\lambda \otimes v$ then we say that $\lambda$ is a max-plus-algebraic eigenvalue of $A$ and that $v$ is a corresponding max-plus-algebraic eigenvector of $A$.

It can be shown that every matrix $A \in \mathbb{R}_{\varepsilon}^{n \times n}$ has at least 1 and at most $n$ max-plus-algebraic eigenvalues (see, e.g. [1]). In particular, irreducible matrices have only one max-plus-algebraic eigenvalue (see, e.g. [3]). For algorithms to determine max-plus-algebraic eigenvalues and eigenvectors the interested reader is referred to $[1,3,15]$ and the references cited therein.

Theorem 2.4. If $A \in \mathbb{R}_{\varepsilon}^{n \times n}$ is irreducible, then

$\exists k_{0} \in \mathbb{N}$ such that $\forall k \geqslant k_{0}: A^{\otimes^{k+c}}=\lambda^{\otimes^{c}} \otimes A^{\otimes^{k}}$

where $\lambda$ is the (unique) max-plus-algebraic eigenvalue of $A$ and $c$ is the cyclicity of $A$.

Proof. See, e.g. [1, 3, 12]. 
Now we give some extra propositions in connection with the cyclicity of a general matrix and with the integer $k_{0}$ that appears in Theorem 2.4 for a boolean matrix. We shall need these propositions in Section 4. The proofs of these (and related) propositions appear in $[8,9]$.

For the cyclicity of a general matrix we have the following upper bound:

Lemma 2.5. If $A \in \mathbb{R}_{\varepsilon}^{n \times n}$ then we have $c(A) \leqslant$ $\exp (n / \mathrm{e})=\alpha^{n}$ with $\alpha=\mathrm{e}^{1 / \mathrm{e}}$.

For general (possibly not irreducible) boolean matrices we can improve the result of Theorem 2.4 by giving an upper bound for the integer $k_{0}$ :

Theorem 2.6. Let $A \in \mathbb{B}^{n \times n}$ and let $c$ be the cyclicity of $A$. We have

$A^{\otimes^{k+c}}=A^{\otimes^{k}}$ for all $k \geqslant 2 n^{2}-3 n+2$.

If $A$ is irreducible then

$A^{\otimes^{k+c}}=A^{\otimes^{k}} \quad$ for all $k \geqslant n^{2}-2 n+2$.

It is easy to verify that the max-plus-algebraic eigenvalue of a max-plus-algebraic boolean matrix is either 0 or $\varepsilon$. That is why $\lambda$ does not appear in Theorem 2.6. The extension of Theorem 2.6 to general matrices with entries in $\mathbb{R}_{\varepsilon}$ is a topic of current research. The following example shows that - in contrast to boolean matrices, where the upper bound for the integer $k_{0}$ of Theorem 2.4 only depends on the size of the matrix - for a general matrix $A$ with entries in $\mathbb{R}_{\varepsilon}$ an upper bound for $k_{0}$ also depends on the range and resolution (i.e., on the size of the representation) of the non- $\varepsilon$ entries of $A$.

Example 2.7. Let $N \in \mathbb{N}$ and consider

$A(N)=\left[\begin{array}{cc}-1 & -N \\ 0 & 0\end{array}\right]$.

The matrix $A(N)$ is irreducible and has cyclicity 1 and max-plus-algebraic eigenvalue 0 . We have

$(A(N))^{\otimes^{k}}=\left[\begin{array}{cc}\max (-k,-N) & -N \\ 0 & 0\end{array}\right]$

for each $k \in \mathbb{N}_{0}$. This implies that the smallest integer $k_{0}$ for which Eq. (3) holds, is given by $k_{0}=N$, i.e., $k_{0}$ depends on the range of the non- $\varepsilon$ entries of $A(N)$.
A similar example can be found in ([1], p. 152). This example shows that in general $k_{0}$ depends on the resolution of the non- $\varepsilon$ entries of the matrix $A$.

\section{Max-plus-algebraic system theory}

\subsection{State space models and impulse responses}

In $[1,3,4]$ it has been shown that there is a class of discrete event systems (DESs) that can be modeled by a max-plus-algebraic model of the following form:

$x(k+1)=A \otimes x(k) \oplus B \otimes u(k)$,

$y(k)=C \otimes x(k)$.

The vector $x$ represents the state, $u$ is the input vector and $y$ the output vector of the system. For a manufacturing system, $u(k)$ would typically represent the time instants at which raw material is fed to the system for the $(k-1)$ th time, $x(k)$ the time instants at which the machines start processing the $k$ th batch of intermediate products, and $y(k)$ the time instants at which the $k$ th batch of finished products leaves the system. A DES that can be modeled by Eqs. (4) and (5) will be called a max-linear time-invariant DES.

The number of components of the state vector $x$ will be called the order of the state space model. We shall characterize a model of the form (4) and (5) by the triple $(A, B, C)$ of system matrices. A system with one input and one output is called a single-input singleoutput (SISO) system. A system with more than one input and more than one output is called a multi-input multi-output (MIMO) system.

Let $i \in\{1,2, \ldots, m\}$. A max-plus-algebraic unit impulse is a sequence $\left\{e_{k}\right\}_{k=0}^{\infty}$ defined by

$e_{k}= \begin{cases}0 & \text { if } k=0, \\ \varepsilon & \text { if } k=1,2, \ldots .\end{cases}$

If we apply a max-plus-algebraic unit impulse to the $i$ th input of the system, and if we assume $x(0)=\varepsilon_{n \times 1}$, then we get

$y(k)=C \otimes A^{\otimes^{k-1}} \otimes B_{., i} \quad$ for $k=1,2,3, \ldots$

as the output of the DES. Note that $y(k)$ corresponds to the $i$ th column of the matrix $G_{k-1} \stackrel{\text { def }}{=} C \otimes A^{\otimes^{k-1}} \otimes B$ for $k=1,2,3, \ldots$ The sequence $\left\{G_{k}\right\}_{k=0}^{\infty}$ is called the impulse response of the DES, and the $G_{k}$ 's are called the impulse response matrices. 
The impulse response of a max-linear time-invariant DES can be characterized by the following theorem:

Theorem 3.1. If $\left\{G_{k}\right\}_{k=0}^{\infty}$ is the impulse response of a max-linear time-invariant DES with $m$ inputs and l outputs then

$$
\begin{aligned}
& \forall i \in\{1,2, \ldots, l\}, \forall j \in\{1,2, \ldots, m\}, \exists c \in \mathbb{N}_{0}, \\
& \exists \lambda_{1}, \lambda_{2}, \ldots, \lambda_{c} \in \mathbb{R}_{\varepsilon}, \exists k_{0} \in \mathbb{N} \text { such that } \forall k \in \mathbb{N}: \\
& \quad\left(G_{k_{0}+k c+c+s-1}\right)_{i j}=\lambda_{s}^{\otimes^{c}} \otimes\left(G_{k_{0}+k c+s-1}\right)_{i j} \\
& \quad \text { for } s=1,2, \ldots, c .
\end{aligned}
$$

Proof. This is a direct consequence of, e.g., Corollary 1.1 .9 of ([11], p. 166) or of Proposition 1.2 .2 of [12].

If a sequence $G=\left\{G_{k}\right\}_{k=0}^{\infty}$ exhibits a behavior of the form (6) then we say that the sequence $G$ is ultimately periodic. If $G=\left\{G_{k}\right\}_{k=0}^{\infty}$ is an ultimately periodic sequence then the smallest possible $c$ for which (6) holds is called the period of $G$.

Proposition 3.2. A sequence $G=\left\{G_{k}\right\}_{k=0}^{\infty}$ with $G_{k} \in \mathbb{R}_{\varepsilon}^{l \times m}$ for all $k$ is the impulse response of $a$ max-linear time-invariant DES if and only if it is an ultimately periodic sequence.

Proof. A proof of this proposition for SISO systems can be found in $[1,11,12]$. For MIMO systems the "only if" part corresponds to Theorem 3.1. To prove the "if" part for MIMO systems we consider each sequence $\left\{\left(G_{k}\right)_{i j}\right\}_{k=0}^{\infty}$ separately; since such a sequence corresponds to a SISO system, we can apply the first part of this proof and afterwards merge all SISO systems into one large MIMO system (see also [5]).

Based on Theorem 3.1 we now introduce a new concept, the so-called canonical representation of the impulse response of a max-linear time-invariant DES or - which is equivalent - of an ultimately periodic sequence. We shall only do this for impulse responses of SISO systems. The extension to MIMO systems is straightforward. The goal of introducing this canonical representation is to get a concise, unique representation of an ultimately periodic sequence. Consider an ultimately periodic sequence of real numbers $g=\left\{g_{k}\right\}_{k=0}^{\infty}$. First we determine the smallest possible $c \in \mathbb{N}_{0}$ for which (6) holds. The $\lambda_{s}$ 's are then defined uniquely ${ }^{3}$ (up to a circular permutation of the indices). Next, we determine the smallest possible $k_{0} \in \mathbb{N}$ such that (6) holds for all $k \geqslant 0$. Now we can uniquely represent the sequence $g$ by the $\left(k_{0}+2 c+1\right)$-tuple $\left(c, \lambda_{1}, \lambda_{2}, \ldots, \lambda_{c}, g_{0}, g_{1}, \ldots, g_{k_{0}+c-1}\right)$. The subsequence $g_{0}, g_{1}, \ldots, g_{k_{0}-1}$ will be called the transient part of $g$.

Example 3.3. Consider the sequence $g=0,0,0,0,0$, $1,0,2,0,3,0,4,0,5, \ldots$. This is an ultimately periodic sequence with period $c=2, \lambda_{1}=0, \lambda_{2}=1$ and $k_{0}=2$. The transient part of $g$ is the subsequence $g_{0}, g_{1}=0,0$. The canonical representation of the sequence $g$ is given by $(2,0,1,0,0,0,0)$.

\subsection{The minimal state space realization problem}

If $G=\left\{G_{k}\right\}_{k=0}^{\infty}$ is an ultimately periodic sequence with $G_{k} \in \mathbb{R}_{\varepsilon}^{l \times m}$ for all $k$, then it follows from Proposition 3.2 that $G$ is the impulse response of a max-linear time-invariant DES with $m$ inputs and $l$ outputs. Now consider the following problem:

Given an ultimately periodic sequence $G=$ $\left\{G_{k}\right\}_{k=0}^{\infty}$ with $G_{k} \in \mathbb{R}_{\varepsilon}^{l \times m}$ for all $k$ and an integer $r$, find, if possible, matrices $A \in \mathbb{R}_{\varepsilon}^{r \times r}, B \in \mathbb{R}_{\varepsilon}^{r \times m}$ and $C \in \mathbb{R}_{\varepsilon}^{l \times r}$ such that $(A, B, C)$ is a realization of $G$, i.e., $G_{k}=C \otimes A^{\otimes^{k}} \otimes B$ for all $k \in \mathbb{N}$.

This problem is called the state space realization problem. If we make $r$ as small as possible, then the problem is called the minimal state space realization problem and the resulting value of $r$ is called the minimal system order.

The minimal state space realization problem for max-linear time-invariant DESs has been studied by many authors and for some very specific cases the problem has been solved (see [7, 13, 16, 17]). However, at present it is still an open problem whether there exist tractable methods to solve the general minimal state space realization problem.

\subsection{The minimal system order}

If $G=\left\{G_{k}\right\}_{k=0}^{\infty}$ is a sequence with $G_{k} \in \mathbb{R}_{\varepsilon}^{l \times r}$ for all $k$, then we define the (semi-infinite) block Hankel

\footnotetext{
${ }^{3}$ Provided that for a subsequence of the form $\varepsilon, \varepsilon, \varepsilon, \ldots$, we take $\lambda_{s}$ equal to $\varepsilon$.
} 
matrix

$H(G) \stackrel{\text { def }}{=}\left[\begin{array}{cccc}G_{0} & G_{1} & G_{2} & \ldots \\ G_{1} & G_{2} & G_{3} & \ldots \\ G_{2} & G_{3} & G_{4} & \ldots \\ \vdots & \vdots & \vdots & \ddots\end{array}\right]$.

The following proposition is a generalization to the MIMO case of Proposition 2.3.1 of ([11], p. 175). It is also an adaptation to max-linear systems of a similar theorem for nonnegative linear systems ([19], Theorem 5.4.10). ${ }^{4}$

Proposition 3.4. Let $G=\{G\}_{k=0}^{\infty}$ be the impulse response of a max-linear time-invariant DES with $m$ inputs and $l$ outputs. Let $n$ be the smallest integer for which there exist matrices $A \in \mathbb{R}_{\varepsilon}^{n \times n}, U \in \mathbb{R}_{\varepsilon}^{\infty \times n}$ and $V \in \mathbb{R}_{\varepsilon}^{n \times \infty}$ such that

$H(G)=U \otimes V \quad$ and $\quad U \otimes A=\bar{U}$,

where $\bar{U}$ is the matrix obtained by removing the first $l$ rows of $U$. Then $n$ is equal to the minimal system order.

Proof. Let $n_{\min }$ be the minimal system order of the given system and let the triple $\left(A_{\min }, B_{\min }, C_{\min }\right)$ be a minimal state space realization of $G$. If we define

$$
\begin{aligned}
U_{\min } & =\left[\begin{array}{c}
C_{\min } \\
C_{\min } \otimes A_{\min } \\
C_{\min } \otimes A_{\min }^{\otimes^{2}} \\
\vdots
\end{array}\right] \text { and } \\
V_{\min } & =\left[\begin{array}{ll}
B_{\min } & \left.A_{\min } \otimes B_{\min } A_{\min }^{\otimes^{2}} \otimes B_{\min } \cdots\right],
\end{array}\right.
\end{aligned}
$$

then it is easy to verify that $U_{\min } \otimes V_{\min }=H(G)$ and $U_{\min } \otimes A_{\min }=\bar{U}_{\text {min }}$. This implies that $n \leqslant n_{\text {min }}$.

Define $\alpha_{k}=\{k l+1, k l+2, \ldots, k l+l\}$ and $\beta_{k}=$ $\{k m+1, k m+2, \ldots, k m+m\}$ for $k=0,1,2, \ldots$ Define $C=U_{\alpha_{0},}$. and $B=V_{\text {., } \beta_{0}}$. Now we prove by induction that $U_{\alpha_{k},}=C \otimes A^{\otimes^{k}}$ for $k=0,1,2, \ldots$.

For $k=0$ we have $U_{\alpha_{0}, .}=C=C \otimes A^{\otimes^{0}}$. Now we assume that $U_{\alpha_{k}, .}=C \otimes A^{\otimes^{k}}$ and we prove that $U_{\alpha_{k+1},}=$ $C \otimes A^{\otimes^{k+1}}$. Since $U \otimes A=\bar{U}$, we have $U_{\alpha_{k+1}, .}=U_{\alpha_{k}, .} \otimes$ $A=C \otimes A^{\otimes^{k}} \otimes A=C \otimes A^{\otimes^{k+1}}$. Since $U \otimes V=H(G)$ we have $C \otimes A^{\otimes^{k}} \otimes B=U_{\alpha_{k}, .} \otimes V_{\text {., } \beta_{0}}=(H(G))_{\alpha_{k}, \beta_{0}}=$

\footnotetext{
${ }^{4}$ Recall that in Remark 2.1 the relation between max-plus algebra and (nonnegative) conventional algebra has been shown. Based on this relation some results from system theory for nonnegative systems can be translated to max-plus-algebraic system theory and vice versa.
}

$G_{k}$. Hence, the triple $(A, B, C)$ is a state space realization of $G$. This implies that $n \geqslant n_{\min }$. Since $n \leqslant n_{\min }$ and $n \geqslant n_{\min }$, we have $n=n_{\min }$.

Definition 3.5 (Max-plus-algebraic Schein rank [11]). Let $A \in \mathbb{R}_{\varepsilon}^{m \times n}$ with $A \neq \varepsilon_{m \times n}$. The smallest integer $r$ for which there exist matrices $U \in \mathbb{R}_{\varepsilon}^{m \times r}$ and $V \in \mathbb{R}_{\varepsilon}^{r \times n}$ such that $A=U \otimes V$ is called the max-plus-algebraic Schein rank of $A$ and it is denoted by $\operatorname{rank}_{\oplus \text {, Schein }}(A)$. By definition we have $\operatorname{rank}_{\oplus, \text { Schein }}(\varepsilon)=0$.

Proposition 3.4 implies that the max-plus-algebraic Schein rank of $H(G)$ is a lower bound for the minimal system order. However, the following theorem shows that, unless $\mathrm{P}=\mathrm{NP}$, this lower bound cannot be computed in a number of operations that increases polynomially with the size of $H(G)$. This remains so even when $H(G)$ is a boolean matrix. (For basic definitions and more information on NP-completeness the reader is referred to [10].)

Theorem 3.6. Determining the max-plus-algebraic Schein rank of a max-plus-algebraic boolean matrix is an NP-hard problem.

Proof. This proof is based on [14]. If $A$ is a boolean matrix then the transition graph of $A$ will be denoted by $\mathscr{G}_{A}$. From ([18], Remark 6.7) it follows that the max-plus-algebraic Schein rank of $A$ is equal to the minimum number of complete bipartite subgraphs of $\mathscr{G}_{A}$ the union of which includes all edges of $\mathscr{G}_{A}$. Indeed, if we consider the incidence matrix of a complete bipartite subgraph, then all the entries of this matrix are equal to 0 . On the other hand, the equation $U \otimes V=A$ can be rewritten as

$$
\bigoplus_{i=1}^{r} U_{., i} \otimes V_{i, .}=A .
$$

It is easy to verify that if $u, v \in \mathbb{B}^{r}$, then the 0 entries of the matrix $u \otimes v^{\mathrm{T}}$ form a submatrix of $u \otimes v^{\mathrm{T}}$. This submatrix corresponds to a complete bipartite subgraph of the transition graph of $u \otimes v^{\mathrm{T}}$. So determining the minimal integer $r$ for which Eq. (7) holds, is equivalent to determining the minimal number of complete bipartite subgraphs of $\mathscr{G}_{A}$ the union of which includes all edges of $\mathscr{G}_{A}$.

Now consider the decision problem that corresponds to the problem of covering a bipartite graph by 
complete bipartite subgraphs (problem GT18 of [10]):

Instance: Bipartite graph $\mathscr{G}$ with a set of vertices $V$ and a set of edges $E$, and a positive integer $K \leqslant \# E$.

Question: Are there $k \leqslant K$ subsets $V_{1}, V_{2}, \ldots, V_{k}$ of $V$ such that each $V_{i}$ induces a complete bipartite subgraph of $G$ and such that for each edge $\{u, v\} \in E$ there is some $V_{i}$ that contains both $u$ and $v$ ?

Since this decision problem is NP-complete $[10,18]$, the problem of determining the minimum number of complete bipartite subgraphs whose union includes all of the edges of a bipartite graph is NP-hard. As a consequence the problem of determining the max-plusalgebraic Schein rank of a max-plus-algebraic boolean matrix is an NP-hard problem.

An upper bound for the minimal system order is given in $[11,12]$ (see also [6]). Note that at present there do not exist efficient (i.e., polynomial time) algorithms to compute a non-trivial lower bound for the minimal system order for a given ultimately periodic sequence.

Since the general minimal realization problem is still an open problem, we consider a simplified version of this problem in the next section.

\section{The boolean minimal realization problem}

A max-linear time-invariant DES for which all the entries of all the impulse response matrices belong to $\mathbb{B}=\{0, \varepsilon\}$ is called a boolean max-linear timeinvariant $D E S$. It is easy to verify that if we have an $r$ th order state space realization $(A, B, C)$ of a boolean max-linear time-invariant DES where the entries of $A, B, C$ belong to $\mathbb{R}_{\varepsilon}$, then there also exists an $r$ th order state space realization $(\tilde{A}, \tilde{B}, \tilde{C})$ such that the entries of $\tilde{A}, \tilde{B}$ and $\tilde{C}$ belong to $\mathbb{B}$.

\subsection{Comparing boolean impulse responses}

The following corollaries are direct consequences of Theorem 2.6.

Corollary 4.1. Consider a boolean max-linear timeinvariant DES with minimal system order $n$ and impulse response $G=\left\{G_{k}\right\}_{k=0}^{\infty}$. Let $c$ be the period of $G$. Then we have

$G_{k+c}=G_{k}$ for all $k \geqslant 2 n^{2}-3 n+2$.
Corollary 4.2. Let $G=\left\{G_{k}\right\}_{k=0}^{\infty}$ and $F=\left\{F_{k}\right\}_{k=0}^{\infty}$ be impulse responses of boolean max-linear timeinvariant DESs with minimal system order less than or equal to $n$. Let $c$ be the maximum of the period of $G$ and the period of $F$. If $G_{k}=F_{k}$ for $k=0,1, \ldots, 2 n^{2}-3 n+1+c$ then $G_{k}=F_{k}$ for all $k \in \mathbb{N}$.

The last corollary gives an explicit upper bound on the number of terms that two impulse responses of boolean max-linear time-invariant DESs should have in common in order to coincide completely.

\subsection{A lower bound for the minimal system order}

Let $G=\left\{G_{k}\right\}_{k=0}^{\infty}$ be the impulse response of a boolean max-linear time-invariant DES. From Proposition 3.4 it follows that the max-plus-algebraic Schein of the matrix $H(G)$ is a lower bound for the minimal system order. From Theorem 3.6 it follows that, unless $\mathrm{P}=\mathrm{NP}$, this lower bound cannot be computed efficiently. However, for a boolean impulse response the following lemma provides an easily computable lower bound for the minimal system order:

Lemma 4.3. Consider a boolean max-linear timeinvariant DES with minimal system order $n$ and impulse response $G=\left\{G_{k}\right\}_{k=0}^{\infty}$. Let $c$ be the period of $G$. Let $L$ be the length of the transient part of the impulse response, i.e., $L$ is equal to the smallest integer $K$ for which we have $G_{k+c}=G_{k}$ for all $k \geqslant K$. If $L \geqslant 2$ then

$n \geqslant \frac{3+\sqrt{8 L-7}}{4}$

Proof. From Corollary 4.1 it follows that

$L \leqslant 2 n^{2}-3 n+2$.

If is easy to verify that this condition holds for every $n \in \mathbb{N}$ if $L=0$ or if $L=1$. So from now on we assume that $L \geqslant 2$. The zeros of the function $f$ defined by $f(n)=2 n^{2}-3 n+2-L$ are $n_{1}=\frac{1}{4}(3+\sqrt{8 L-7})$ and $n_{2}=\frac{1}{4}(3-\sqrt{8 L-7})$. Since $n_{2} \leqslant 0$ if $L \geqslant 2$ and since $n$ is always positive, the function $f$ will be nonnegative if $n \geqslant n_{1}$. Hence, condition (8) will only be satisfied if $n \geqslant n_{1}$. 


\subsection{The complexity of the boolean minimal realiza- tion problem}

In this section we consider the following two problems:

- the boolean realization decision problem (BRDP): Given an ultimately periodic sequence $G=\left\{G_{k}\right\}_{k=0}^{\infty}$ with $G_{k} \in \mathbb{B}^{l \times m}$ in its canonical representation and an integer $r$, does there exist an $r$ th order boolean state space realization of $G$ ? This problem will be denoted by $\operatorname{BRDP}(G, r)$.

- the boolean minimal realization problem (BMRP): Given an ultimately periodic sequence $G=\left\{G_{k}\right\}_{k=0}^{\infty}$ with $G_{k} \in \mathbb{B}^{l \times m}$ in its canonical representation, compute a minimal state space realization of $G$. This problem will be denoted by $\operatorname{BMRP}(G)$.

Proposition 4.4. Let $G=\left\{G_{k}\right\}_{k=0}^{\infty}$ be an ultimately periodic sequence with $G_{k} \in \mathbb{B}^{l \times m}$ and let $r \in \mathbb{N}$. The problem $\operatorname{BRDP}(G, r)$ is decidable using a finite number of elementary operations (such as addition, subtraction, multiplication, division, maximum, minimum and comparison).

Proof. Since $G$ is an ultimately periodic sequence, it corresponds to the impulse response of a boolean maxlinear time-invariant DES. Let $n$ be the minimal system order of this system. From ([6], Proposition A.6) it follows that an upper bound $n_{\mathrm{u}}$ for $n$ can be computed in a finite number of steps. If $r \geqslant n_{\mathrm{u}}$ then there exists an $r$ th order state space realization of $G$ and then the answer to the $\operatorname{BRDP}(G, r)$ is affirmative.

From now on we assume that $r \leqslant n_{\mathrm{u}}$. Let $c$ be the period of $G$. Define $K=2 n_{\mathrm{u}}^{2}-3 n_{\mathrm{u}}+1+c$. If we have an $r$ th order state space realization characterized by the triple of system matrices $(A, B, C)$ and if $C \otimes A^{\otimes^{k}} \otimes B=G_{k}$ for all $k \leqslant K$ then it follows from Corollary 4.2 that $(A, B, C)$ is an $r$ th order state space realization of $G$.

This implies that the $\operatorname{BRDP}(G, r)$ is equivalent to checking whether or not the following system of equations has a solution:

$C \otimes A^{\otimes^{k}} \otimes B=G_{k} \quad$ for $k=0,1, \ldots, K$,

with $A \in \mathbb{B}^{r \times r}, B \in \mathbb{B}^{r \times m}$ and $C \in \mathbb{B}^{l \times r}$. Since

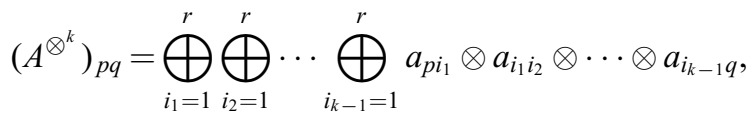

Eq. (9) can be rewritten as

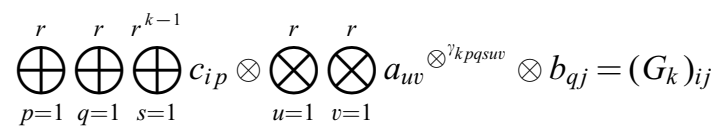

for $i=1,2, \ldots, l, j=1,2, \ldots, m$ and $k=0,1, \ldots, K$, where $\gamma_{k p q s u v}$ is the number of times that $a_{u v}$ appears in the $s$ th term of $\left(A^{\otimes^{k}}\right)_{p q}$. Note that if $a_{u v}$ does not appear in that term we have $\gamma_{k p q s u v}=0$ since $a^{\otimes^{0}}=0 \cdot a=0$. If we put the entries of $A, B$ and $C$ in one large column vector $x$ of length $L=(r+m+l) r$, if we put the entries of the $\left(G_{k}\right)_{i j}$ 's in one large column vector $d$ of length $M=\operatorname{lm}(K+1)$ and if we reformulate everything in conventional algebra, Eq. (10) is an equation of the form

$\max _{i}\left(\alpha_{k i 1} x_{1}+\alpha_{k i 2} x_{2}+\cdots+\alpha_{k i L} x_{L}\right)=d_{k}$.

The system of equation (11) with $k=0,1, \ldots, M$ can be solved using an exhaustive search method: First we select for the first equation a term for which the maximum is reached, and we eliminate a variable if possible. Then we select for the second equation a term for which the maximum is reached, and so on, until we either find a solution or reach an inconsistent system of equations. In the latter case we backtrack and select another candidate for the maximizing term in the equation where a last choice was made. This continues until we either find a solution (which yields an $r$ th order state space realization of $G$ ), or have exhausted all possible choices, in which case the system cannot be solved (which implies that no $r$ th order state space realization of $G$ exists). Hence, we can give an answer to $\operatorname{BRDP}(G, r)$ using a finite number of elementary operations.

Remark 4.5. A similar reasoning can be used to show that the general realization decision problem is also decidable provided that we can give an a priori upper bound for the number of terms $K$ in the system (9).

In the formulation of Proposition 4.4 we have used the concept "decidability" in a rather loose and informal way. However, it can be verified that our use of decidability corresponds to the formal concept of decidability in the Turing machine sense.

Proposition 4.6. Let $G=\left\{G_{k}\right\}_{k=0}^{\infty}$ be an ultimately periodic sequence with $G_{k} \in \mathbb{B}^{l \times m}$ for all $k$. Let $n_{\mathrm{u}}$ be 
an upper bound ${ }^{5}$ for the minimal system order of the max-linear time-invariant DES the impulse response of which coincides with $G$. Then $\operatorname{BMRP}(G)$ can be solved in a number of elementary operations that is bounded from above by the function $f$ defined by

$$
\begin{aligned}
f\left(n_{\mathrm{u}}, l, m\right)= & 2 m\left\lceil 2 n_{\mathrm{u}}^{2}-3 n_{\mathrm{u}}+2+\exp \left(\frac{n_{\mathrm{u}}}{e}\right)\right\rceil \\
& \times \sum_{r=1}^{n_{\mathrm{u}}} r(l+r) 2^{r^{2}+r(m+l)} .
\end{aligned}
$$

Furthermore, $f\left(n_{\mathrm{u}}, l, m\right) \leqslant \gamma^{n_{\mathrm{u}}^{2}}$ with $\gamma=3^{m+l+3}$.

Proof. Since $G$ is ultimately periodic it corresponds to the impulse response of a max-linear time-invariant DES. Furthermore, since all the entries of the $G_{k}$ 's are in $\mathbb{B}, G$ also corresponds to the impulse response of a boolean max-linear time-invariant DES.

Assume that the minimal system order of the boolean max-linear time-invariant DES we are looking for is equal to $n$. Let $n_{1}$ be a lower bound for the minimal system order (that is, e.g., obtained by using Lemma 4.3).

If $c$ is the period of $G$, then $c \leqslant \exp (n / \mathrm{e})$ by Lemma 2.5. Hence, $c \leqslant \exp \left(n_{\mathrm{u}} / \mathrm{e}\right)$. Define $K=$ $\left\lceil 2 n_{\mathrm{u}}^{2}-3 n_{\mathrm{u}}+2+\exp \left(n_{\mathrm{u}} / \mathrm{e}\right)\right\rceil$. If we have a sequence $F=\left\{F_{k}\right\}_{k=0}^{\infty}$ that is the impulse response of an $r$ th order boolean max-linear time-invariant DES with $r \leqslant n_{\mathrm{u}}$, then by Corollary 4.2 it suffices to check whether the first $K$ terms of $F$ and $G$ are equal in order to decide whether $F$ and $G$ coincide.

Now we can apply the following procedure which is combination of an incremental search procedure ${ }^{6}$ (for the system order) combined with an enumerative procedure (for the entries of the system matrices). We start with a guess $r$ for the minimal system order that is equal to $n_{1}$. Then we consider all possible triples $(A, B, C)$ with $A \in \mathbb{B}^{r \times r}, B \in \mathbb{B}^{r \times m}$ and $C \in \mathbb{B}^{l \times r}$. For each triple we consider the finite sequence $F=\left\{C \otimes A^{\otimes^{k}} \otimes B\right\}_{k=0}^{K-1}$. If the terms of this sequence are equal to the first $K$ terms of $G$, then the triple $(A, B, C)$ is a minimal state space realization of $G$ and $r$ is the minimal system order. Otherwise, we consider the next triple $(A, B, C)$. Note that the number of triples that should be considered is less than or equal to $2^{r^{2}+r(m+l)}$. For each triple $(A, B, C)$ we have to compute at most $K$ terms of the sequence $F$ and

\footnotetext{
${ }^{5}$ See $[6,11,12]$ for a finite upper bound for the minimal system order that can be computed efficiently.

${ }^{6} \mathrm{We}$ could also have used a binary search procedure.
}

compare them with the corresponding term of $G$. It is easy to verify that this can be done using a number of additions or comparisons that is less than or equal to

$$
\begin{aligned}
& K \operatorname{lm}(2 r-1)+(K-1) r m(2 r-1)+K \operatorname{lm} \\
& \quad=K \operatorname{lm}(2 r)+(K-1) r m(2 r-1) \\
& \quad \leqslant K \operatorname{lm} 2 r+K r m 2 r \\
& \quad \leqslant 2 \operatorname{Km} r(r+l) .
\end{aligned}
$$

If all $r$ th order triples have been considered and no state space realization of $G$ has been found yet, we augment $r$ and repeat the procedure described above.

Since $n_{\mathrm{u}}$ is an upper bound for the minimal system order, this procedure will ultimately lead to a minimal state space realization of $G$. Note that in the worst case $r$ ranges from 1 to $n_{\mathrm{u}}$.

Hence, the number of elementary operations that is needed to solve $\operatorname{BMRP}(G)$ in bounded from above by the function $f$ defined by Eq. (12).

Furthermore, it can be verified that $f\left(n_{\mathrm{u}}, l, m\right) \leqslant \gamma^{n_{\mathrm{u}}^{2}}$ for all $n_{\mathrm{u}}, l, m \in \mathbb{N}_{0}$.

It is still an open problem whether there exist polynomial time algorithms to solve the BRDP and the BMRP.

\section{Conclusions}

In this paper we have considered the minimal state space realization problem for max-linear timeinvariant discrete event systems (DESs). We have derived a lower bound for the minimal system order and discussed the computational complexity of computing this lower bound. We have also introduced a canonical representation of the impulse response of a max-linear time-invariant DES. Next we have directed our attention to the boolean minimal realization problem. We have shown that this problem can be solved in a number of operations that is bounded from above by an exponential of the square of the minimal system order. We have also derived an efficiently computable lower bound for the minimal system order.

In our future research we hope to extend some of the results of this paper to general max-linear timeinvariant DESs. 


\section{Acknowledgements}

The algorithm used in the proof of Proposition 4.4 was suggested to the first author by S. Gaubert. The algorithm can be thought of as a Tarski-Seidenberg elimination method for the max-plus algebra. Bart De Schutter is a senior research assistant with the F.W.O. (Fund for Scientific Research-Flanders). Bart De Moor is a research associate with the F.W.O. This research was sponsored by the Concerted Action Project of the Flemish Community, entitled "Modelbased Information Processing Systems" (GOAMIPS), by the Belgian program on interuniversity attraction poles (IUAP P4-02 and IUAP P4-24), by the ALAPEDES project of the European Community Training and Mobility of Researchers Program, and by the European Commission Human Capital and Mobility Network SIMONET ("System Identification and Modelling Network").

\section{References}

[1] F. Baccelli, G. Cohen, G.J. Olsder, J.P. Quadrat, Synchronization and Linearity, Wiley, New York, 1992.

[2] G. Cohen, D. Dubois, J.P. Quadrat, M. Viot, Analyse du comportement périodique de systèmes de production par la théorie des dioïdes, Tech. Rep. 191, INRIA, Le Chesnay, France, February 1983.

[3] G. Cohen, D. Dubois, J.P. Quadrat, M. Viot, A linearsystem-theoretic view of discrete-event processes and its use for performance evaluation in manufacturing, IEEE Trans. Automat. Control 30 (3) (1985) 210-220.

[4] R.A. Cuninghame-Green, Minimax Algebra, Lecture Notes in Economics and Mathematical Systems, vol. 166, Springer, Berlin, 1979.

[5] B. De Schutter, Max-algebraic system theory for discrete event systems, Ph.D. Thesis, Faculty of Applied Sciences, K.U. Leuven, Leuven, Belgium, 1996.

[6] B. De Schutter, V. Blondel, R. de Vries, B. De Moor, On the boolean minimal realization problem in the max-plus algebra: Addendum, Tech. Rep. 97-68a, ESAT-SISTA, K.U. Leuven, Leuven, Belgium, December 1997.
[7] B. De Schutter, B. De Moor, Minimal realization in the max algebra is an extended linear complementarity problem, Systems Control Lett. 25 (2) (1995) 103-111.

[8] B. De Schutter, B. De Moor, On the sequence of consecutive powers of a matrix in a boolean algebra, Tech. Rep. 97-67, ESAT-SISTA, K.U. Leuven, Leuven, Belgium, April 1998, revised version, submitted for publication.

[9] B. De Schutter, B. De Moor, On the ultimate behavior of the sequence of consecutive powers of a matrix in the maxplus algebra, Tech. Rep. 98-32, ESAT-SISTA, K.U. Leuven, Leuven, Belgium, May 1998.

[10] M.R. Garey, D.S. Johnson, Computers and Intractability: A Guide to the Theory of NP-Completeness, Freeman, San Francisco, 1979.

[11] S. Gaubert, Théorie des systèmes linéaires dans les diö̈des, Ph.D. thesis, Ecole Nationale Supérieure des Mines de Paris, France, July 1992.

[12] S. Gaubert, On rational series in one variable over certain dioids, Tech. Rep. 2162, INRIA, Le Chesnay, France, January 1994.

[13] S. Gaubert, P. Butkovič, R. Cuninghame-Green, Minimal $(\max ,+)$ realization of convex sequences, SIAM J. Control Optim. 36 (1) (1998) 137-147.

[14] D.A. Gregory, N.J. Pullman, Semiring rank: Boolean rank and nonnegative rank factorizations, J. Combin. Inform. System Sci. 8 (3) (1983) 223-233.

[15] R.M. Karp, A characterization of the minimum cycle mean in a digraph, Discrete Math. 23 (1978) 309-311.

[16] G.J. Olsder, Some results on the minimal realization of discrete-event dynamic systems, Tech. Rep. 85-35, Delft University of Technology, Faculty of Technical Mathematics and Informatics, Delft, The Netherlands, 1985.

[17] G.J. Olsder, On the characteristic equation and minimal realizations for discrete-event dynamic systems, in: Proc. 7th Internat. Conf. on Analysis and Optimization of Systems, Antibes, France, Lecture Notes in Control and Information Sciences, vol. 83, Springer, Berlin, 1986, pp. 189-201.

[18] J. Orlin, Contentment in graph theory: covering graphs with cliques, Proc. Koninklijke Nederlandse Akademie van Wetenschappen: Series A: Mathematical Sciences, vol. 80, 1977, pp. 406-424.

[19] J.M. van den Hof, System theory and system identification of compartmental systems, Ph.D. thesis, Faculty of Mathematics and Natural Sciences, University of Groningen, Groningen, The Netherlands, November 1996. 